\title{
Airway smooth muscle: an architect of the lung?
}

\author{
E C Jesudason
}

Correspondence to:

Mr E C Jesudason, MRC New Investigator and Consultant

Paediatric Surgeon, Division of Child Health, University of

Liverpool, Alder Hey Children's

Foundation NHS Trust, Institute of Child Health, Eaton Road,

Liverpool L12 2AP, UK;

e.jesudason@liv.ac.uk

Received 1 September 2008 Accepted 31 October 2008

\section{ABSTRACT}

This review examines the rationale for increased study of the prenatal development of airway smooth muscle (ASM). With a critical role in asthma and airway remodelling, ASM has been extensively investigated. Nevertheless, there is a paucity of studies looking at prenatal ASM development and function. A working party report from the National Heart Lung and Blood Institute identified this issue and has recommended further investigation. The impetus for this call stems not only from an appreciation that childhood and adulthood disease may have their origins in fetal life, but also because recent studies indicate that prenatal ASM regulates lung development (via its phasic contractility and growth factor production). Moreover, recognition that phasic ASM contractility is the prenatal norm has raised interest in the idea that postnatal reactive airways disease may likewise be a periodic oscillatory phenomenon. Finally, bronchial thermoplasty represents an exciting new therapy for refractory asthma. However, the mechanism for its effects is unclear. Recent studies show that prenatal ASM contractility is governed by a hierarchy of pacemakers within proximal airways. If such a pacemaker arrangement persists postnatally, it is possible that bronchial thermoplasty achieves its distal airway effects via ablation of controlling proximal pacemaker centres. Hence, study of prenatal ASM may allow us not only to develop ways to stimulate prenatal lung growth, but also to understand and develop new therapies for reactive airways disease.

Airway smooth muscle (ASM) has memorably been described as "the appendix of the lung". ${ }^{1}$ This epithet has captured the contestable idea that ASM has no physiological purpose and, indeed, only has pathological consequence. Resolving whether ASM has a purpose has been a particular priority for proponents of bronchial thermoplasty (since they seek to ablate ASM). ${ }^{2}$ Opponents, arguing that ASM is useful, have suggested a number of different putative functions ranging from participation in fetal lung growth to optimisation of dead space. $^{3}$ Unfortunately, few of these postulated functions of ASM have been extensively tested. Now, however, the National Heart Lung and Blood Institute (NHLBI) has called for further investigation of prenatal ASM development. ${ }^{4}$ Numerous reasons are likely to underpin such a request; however, it is clear that, relative to its postnatal study, prenatal investigation of ASM has been largely overlooked. The central role of ASM in reactive airways disease, asthma and airway remodelling is well reviewed elsewhere. ${ }^{5}$ This article will discuss what is known about prenatal ASM and how the latest data provide new insights on lung development and postnatal lung disease. Before embarking, it is worth reflecting that the potential fetal origins of adult disease are well described with regard to cardiovascular morbidity and mortality; more recently there has been interest in the early life origins of adult respiratory illnesses such as chronic obstructive pulmonary disease. ${ }^{6}$ Hence, it is both timely and reasonable to investigate the potential contribution of prenatal ASM dysfunction to postnatal reactive airways disease. The latter is one of the commonest chronic illnesses treated in UK primary care and features with greater frequency among those whose lung development is disordered, either by prematurity or significant birth defect (eg, congenital diaphrag-

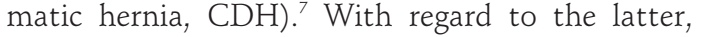
Schuger's group have already demonstrated abnormalities of ASM-related gene expression in hypoplastic lung from patients with $\mathrm{CDH} .{ }^{8}$ Thus, not only has it been suggested that ASM contributes to normal lung development, but abnormalities in its elaboration may have consequences for postnatal pulmonary morbidity. ${ }^{9}$ Finally, it is instructive to consider a teleological argument: several types of diving mammal feature prominent ASM elaboration. Given such mammals appear to have evolved from terrestrial antecedents, it may be harder to argue that ASM is just an evolutionary vestige like the vermiform appendix. Indeed, studies of certain diving mammals have argued that heavy ASM elaboration protects against nitrogen narcosis and decompression sickness by facilitating efficient alveolar and distal airway collapse (and thereby confining residual pulmonary air to the anatomical dead space). ${ }^{10}$ In summary, despite considerable investigation of postnatal ASM, there is still no agreement as to its function. However, of the physiological functions that have been suggested in humans, potential roles in lung development are now being elucidated. ${ }^{11}$ Dismissing ASM as an evolutionary vestige may yet be premature.

\section{WHAT IS KNOWN ABOUT ASM DEVELOPMENT?}

The human lung originates as a diverticulum from the primitive foregut at 3-4 weeks of gestation (approximately day 9.5 and 11 of murine and rat gestation, respectively). Aside from the different number of lobar branches between species, these early events and the signalling that governs them seem to be well conserved between humans and experimental animals. From studies of murine and rat lung development, it is clear that ASM development closely follows the emergence of the definitive lung bud. ${ }^{12}$ Taking alpha smooth muscle actin (SMA) as an early marker of ASM differentiation, SMA is present around the proximal airways by the equivalent of 5- 6 weeks of human gestation. ${ }^{12}$ The origin of such ASM cells has been elucidated in several landmark studies by Schuger 
and recently by Mailleux et al. Schuger's work neatly demonstrated that primitive lung mesenchymal cells retain a generic capacity to differentiate into ASM, and that this pathway of development is facilitated by not only key intercellular signalling (eg, laminin, Rho A) but also by critical changes in mesenchymal cell shape. ${ }^{13-15}$ In particular, it would appear that the ability of the mesenchymal cells to stretch over a surface is important in inducing ASM differentiation. This property is invoked to explain why mesenchymal cell contact with the epithelial basement membrane promotes ASM differentiation (and hence how ASM encircles the airways). Schuger's work has also indicated that the degree of stretch within the developing lung helps determine the degree of ASM differentiation. To test this, she varied the intraluminal fluid content of lung explants using osmotically active dextran. As water is drawn to intraluminal dextran, lung fluid increases with greater stretch of the epithelial basement membrane and attached mesenchymal cells. Depending on the precise dextran concentration used, ASM differentiation increases in response. An opposite effect is noted when the dextran is placed extraluminally to reduce lung liquid-induced airway stretch. ${ }^{8}$ This key body of work has therefore demonstrated the critical importance of mechanical factors such as cell shape, spreading and stretch on ASM elaboration. Moreover, this places ASM cells in an ideal position not only to respond to but, indeed, to generate mechanical forces that can then influence lung growth. That mechanics have a key influence on prenatal lung development is demonstrated from a number of sources (reviewed by Harding and Hooper $\left.{ }^{16}\right)$ :

- Fetal tracheostomy with drainage of lung liquid is associated with hypoplastic lung development.

- Fetal tracheal occlusion (either due to laryngeal atresia or fetal endotracheal balloon placement) is associated with lung distension and overgrowth.

- A fetal space-occupying lesion within the thorax (such as a balloon or surgically created diaphragmatic hernia) is associated with impaired lung development.

- Fetal phrenic nerve section to abolish rhythmic fetal breathing movements is also associated with lung hypoplasia.

- At a cellular level, stretch is a well-recognised regulator of key gene expression and proliferation in pulmonary cells. ${ }^{8}$

In this context, the observation that ASM is not only synthetically but mechanically active from the early stages of lung development has been of increasing interest. ${ }^{12}$ Airway peristalsis (AP) is the spontaneous phasic propagation of waves of ASM contractility that have been observed in a number of species ranging from chicks to humans ${ }^{17-19}$ (see video example at http://ajrcmb.atsjournals.org/content/vol32/issue2/images/data/ 118/DC1/Jesudason_Supplement_001.avi). AP appears in the first trimester and then increases in frequency towards birth. $^{12} 18$ Although this activity was described more than 80 years ago, the purpose of such peristaltic waves has remained obscure. What is clear from our studies and those of others is that these phasic waves of contraction generate not only intraluminal fluid flux, but also periodic distension and relaxation of the end buds of the lung. ${ }^{3}$ Since growth is concentrated at these end buds and stretch critically modulates growth, AP is a good candidate regulator of prenatal pulmonary development. By alternately stimulating or inhibiting such contractility in cultured lung explants, we have shown that the frequency of such airway peristalsis appears to be coupled to lung growth. ${ }^{12}$ Moreover, alternately inhibiting or stimulating lung growth seems to have parallel effects on peristaltic frequency. Recent studies have also shown that fetal tracheal occlusion (an experimental therapy to rescue lung hypoplasia in $\mathrm{CDH}$ ) is associated not only with a rise in intraluminal pulmonary pressures, but also the emergence of trifurcation rather than bifurcation within the airway. ${ }^{20}$ This again suggests that modulating intraluminal pressure by manipulation of peristaltic activity may have significant effects on the morphogenesis of end buds and hence lung development in general.

While Schuger has shown that early pulmonary mesenchymal cells have a generic capacity to differentiate into ASM, it is clear that not all such cells do so. Determining the identity and location of ASM progenitors has become possible through the studies of Mailleux et al. ${ }^{21}$ Indeed, the identification of ASM progenitor cells has been accompanied by significant excitement within the field because it has become clear that such precursor cells are responsible not only for elaboration of mechanically active ASM, but also for furnishing a key growth factor that is essential for respiratory organogenesis in species as diverse as Drosophila, mouse and human. Fibroblast growth factor-10 (FGF10) is required for both limb and lung morphogenesis in mice. ${ }^{22}$ FGF10 -/- null mutants undergo no airway development beyond the carina. Similarly, the Drosophila homologues for FGF10 and its receptor (in mammals, FGFR2IIIb) have been named "branchless" and "breathless" due to the failure of tracheal morphogenesis in mutants lacking either. ${ }^{23}$ Using a serendipitously generated FGF10 Lac Z reporter mouse, Mailleux et al showed that production of essential FGF10 originated from the distal mesenchymal cells that surround the growing end buds of the lung. ${ }^{21}$ Having produced FGF10 (to drive growth), these mesenchymal cells then differentiate into parabronchial ASM. Hence, for a cell that has been described as "frustrated", recent evidence suggests that ASM may have a critical role not only in the biomechanics of lung development (via AP), but also in its biochemical regulation (via FGF10 production).

In addition to the distal ASM progenitors identified by Mallieux et al, a recent study by Shan et $a^{24}$ indicates that a subset of ASM cells originates from proximal lung mesenchyme. This subpopulation may have particular significance given that spontaneous ASM peristalsis appears to be preferentially generated from discrete pacemaker sites within the proximal airway. ${ }^{12}$ In particular, at early stages of development, most waves originate from the proximal right lung. This pacemaker area would seem to dominate the rest of the airway since other areas of the lungs (eg, in the proximal left lung) exhibit faster escape rhythms when the right lung pacemaker is ablated. ${ }^{12}$ Moreover, the right lung pacemaker seems to be autonomous, given that when it is isolated from the rest of the airway its rhythm is unchanged. It should also be noted that these are non-neurogenic pertistaltic waves that are unaffected by tetrodotoxin. ${ }^{3}$ This pacemaker hierarchy within the developing lung appears to change gradually during development; while AP waves originating in the right lung continue to predominate, those originating in the trachea subside as morphogenesis progresses. ${ }^{25}$ In experimental models of lung hypoplasia and $\mathrm{CDH}$, both the calcium signalling that underpins AP and also the spatial organisation of the pacemaker hierarchy are abnormal. ${ }^{25-27}$ Again this work suggests that lung growth and ASM activity (in this case, the spatial orientation of ASM pacemakers) are interrelated. Moreover, if a pacemaker hierarchy is preserved in postnatal lung, we may have a novel explanation for the beneficial effects of bronchial thermoplasty. ${ }^{2}$ The latter will be discussed in greater detail below. 
Figure 1 The fluid pump hypothesis: a spontaneous propagating intercellular airway smooth muscle (ASM) calcium wave (represented by the labelled arrow) precedes each wave of airway peristalsis (not shown). Prenatal lung liquid secretion is led by epithelial chloride secretion. If, as suggested, each ASM calcium wave triggers calcium-activated chloride release, airway peristalsis could provide local pulses of chloride to facilitate lung liquid production.
Proximal

Airway lumen

Distal

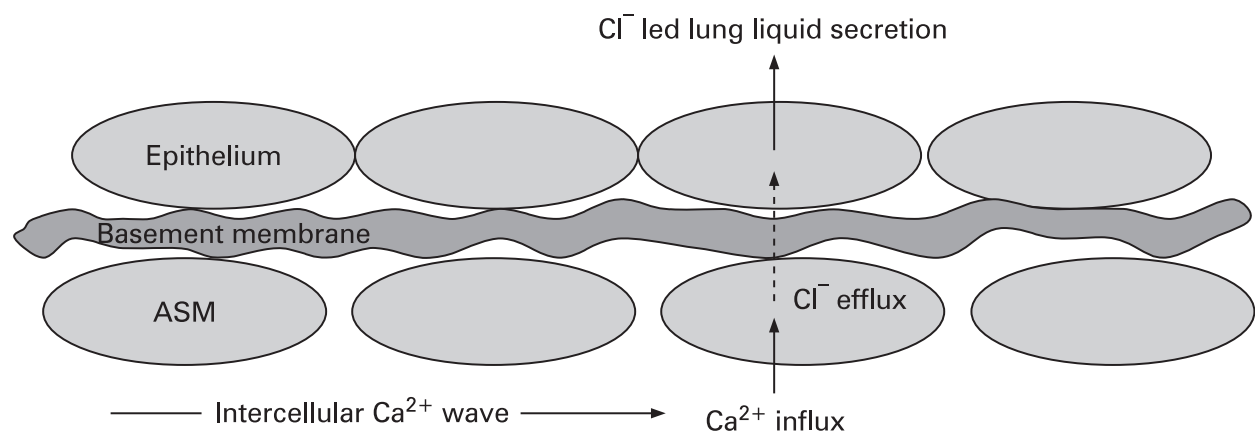

Beyond the mechanical effects achieved by rhythmic ASM contractility, it has been suggested that ASM peristalsis regulates lung liquid production. ${ }^{28}$ Lung fluid secretion before birth is essential for normal pulmonary growth and is thought to occur via epithelial chloride secretion. ${ }^{29}$ ASM may provide pulses of chloride to the adjacent epithelium for lung liquid formation: each wave of prenatal AP is preceded by a spontaneous propagating calcium wave. ${ }^{26}$ Such calcium waves may provoke calcium-activated chloride channels to allow chloride efflux. If such chloride efflux is used by the adjacent epithelium, each intercellular wave of ASM calcium activation generates a pulse of chloride efflux that drives lung liquid secretion (fig 1).

In the next section the translational aspects of this latest research will be discussed along with proposed mechanisms whereby ASM can regulate prenatal lung growth. Finally, the

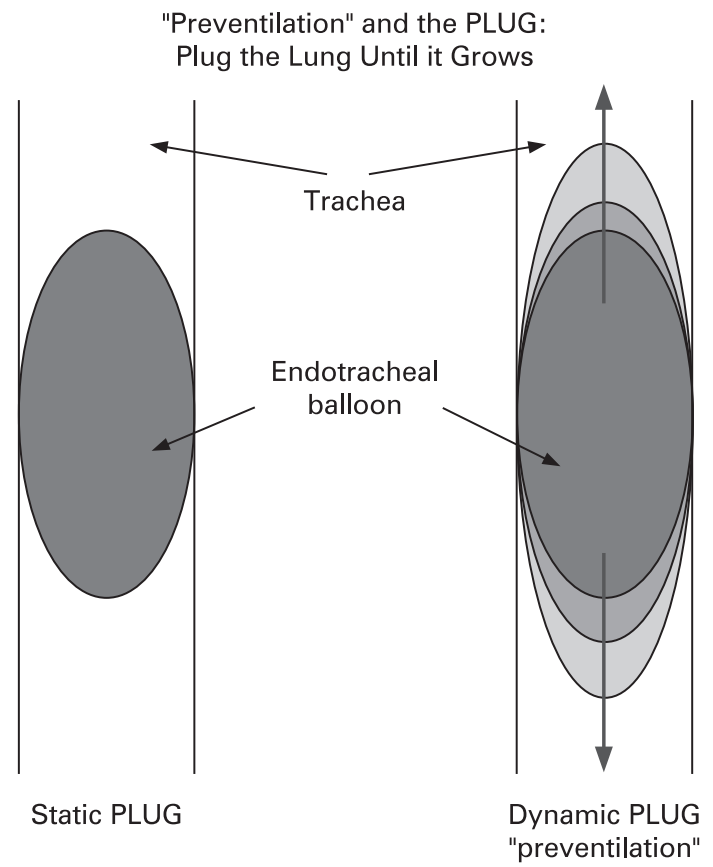

Figure 2 "Preventilation": an illustration of how fetoscopic balloon occlusion of the trachea may be modified by substituting a static "PLUG" (Plug the Lung Until it Grows) for a dynamic pulsatile one. The latter may better preserve the oscillatory mechanics known to influence fetal lung growth and hence improve on the equivocal results achieved to date by fetal tracheal occlusion for lung hypoplasia in congenital diaphragmatic hernia. impact of such understanding on postnatal lung disease will be examined.

\section{TRANSLATIONAL OPPORTUNITIES ARISING FROM NOVEL INSIGHTS INTO ASM DEVELOPMENT}

As previously mentioned, there is growing interest in the early origins of both childhood and adult disease. Perhaps most researched in the cardiovascular system, it is clear that respiratory health may also have many of its determinants in early life. ${ }^{6}$ Recent experimental evidence indicates that ASM and its progenitors regulate not only critical growth factor signalling, but also modulate biomechanics that are central to pulmonary growth. Moreover, if ASM peristalsis regulates prenatal lung development, then we have demonstrated that calcium waves regulate lung morphogenesis. ${ }^{12} 26$

\section{Preventilation and the polyPLUG: alternatives to fetal tracheal occlusion for $\mathrm{CDH}$}

$\mathrm{CDH}$ is a birth defect that is almost as common as cystic fibrosis. It comprises intrathoracic herniation of abdominal viscera, lung hypoplasia and pulmonary hypertension. Together these result in respiratory failure that is chiefly responsible for the mortality rate of approximately $40 \%$. Such has been the difficulty in treating these newborns that fetal surgical approaches have been developed to try to rescue hypoplastic lung growth prenatally. ${ }^{30}$ Based on the observation that congenital laryngeal atresia is associated with huge hyperplastic lungs, fetal tracheal occlusion (by tracheal clipping and later fetoscopic endotracheal balloon placement) has been trialled as a therapy for lung hypoplasia in $\mathrm{CDH}$. However, despite success in animal models, fetal tracheal occlusion (known as the PLUG: Plug the Lung Until it Grows) has yet to show conclusive benefit in humans. ${ }^{30}$ In part this may be due to difficulty in accurately identifying those cases of fetal $\mathrm{CDH}$ that would benefit from such intervention. ${ }^{31}$ However, failure to show benefit may also be due to the type of tracheal occlusion employed; animal studies have suggested that periodic "on and off" PLUG may be better than the one-off "static" tracheal occlusion currently in human practice. ${ }^{32}$ Extending this idea further, it is conceivable that replacing the static PLUG with a dynamic alternative (eg, a pulsatile balloon like an aortic balloon pump that generates a degree of "preventilation") may preserve and/or mimic the oscillatory mechanics (fetal breathing and AP) that characterise normal lung growth (fig 2). Given the fractallike nature of the airways, an alternative possibility is that hypoplastic lung growth may be rescued by multiple transient occlusions of the medium and smaller airways rather than 
depending on tracheal occlusion. Just as hyperplastic lungs in congenital laryngeal atresia illustrated the therapeutic potential of the PLUG, the hyperinflated lungs of patients with chronic asthma show that ASM behaviour alters lung morphology and supports the concept that prenatal bronchospasm may even be a means of augmenting prenatal lung growth (the polyPLUG hypothesis). ${ }^{11}$

\section{Pacemaker Ablation by THermoplasty (PATH) hypothesis}

The potential translational benefits of such new insights into ASM development may be greater still for postnatal lung disease. For example, bronchial thermoplasty is a promising new therapy for refractory asthma. ${ }^{2}$ Aiming to reduce ASM mass (with minimal epithelial injury and scarring), thermal energy from a radiofrequency source is delivered to the airways bronchoscopically. However, it has remained a puzzle how a therapy that is deployed to large and medium-sized airways can effectively modulate a disease thought to involve the small to medium-sized airways. This apparent paradox may be resolved if postnatal ASM contractility is governed by a hierarchy of proximal pulmonary pacemakers that are similar to those already demonstrated within developing lung. ${ }^{12}{ }^{25}$ Hence, thermoplasty would have its effect by ablating the dominant proximal pacemakers with consequent downstream effects on subordinate centres (fig 3). If this is the case, it holds the possibility that thermoplasty may be replaceable by an

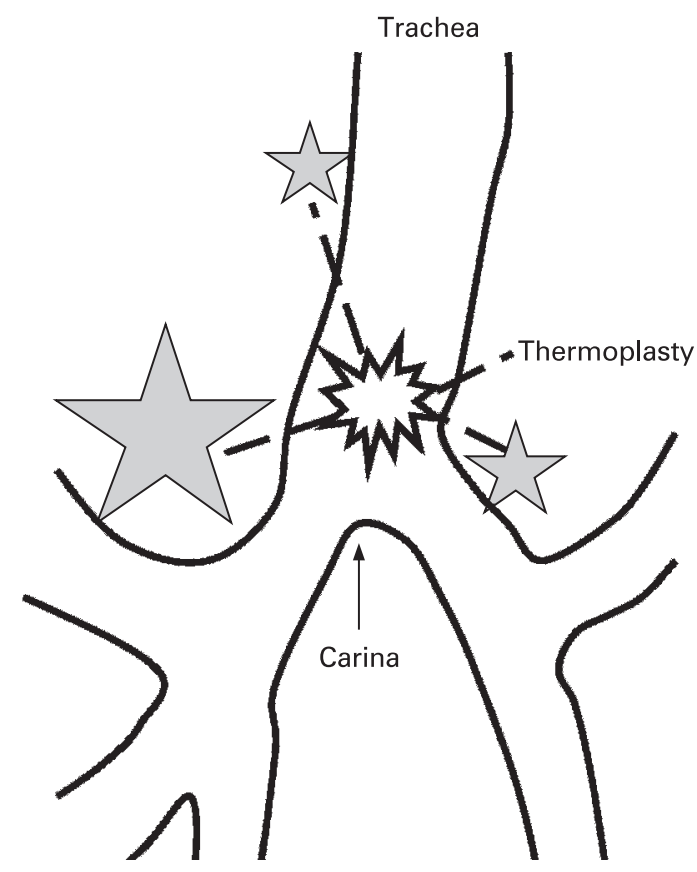

Figure 3 Pacemaker Ablation by THermoplasty (PATH) hypothesis. The apparent benefits of bronchial thermoplasty may be difficult to explain, given that the therapy targets larger airways while asthma is conventionally considered a disease of small and medium-sized airways. A pacemaker hierarchy governs prenatal ASM contractility and is concentrated in the proximal airways. The shaded stars show the early prenatal location of the pacemaker areas. The size of each star approximately represents the frequency of airway peristalsis waves emanating from that site; this therefore illustrates the observed dominance of the right lung in the generation of prenatal airway contractions. Should such proximal pacemakers persist postnatally and similarly regulate distal airway contractility, then their ablation by thermoplasty (represented by the "blast" symbol) may help to explain its effects on the small to medium-sized airways. alternative means to target proximal airway pacemakers and thereby control the activation and/or quiescence of distal ASM. For instance, Shan et $a l^{24}$ suggest that a subset of ASM progenitors arises proximally; we can postulate that this proximally-derived ASM subpopulation plays a key role in the observed pacemaking of proximal ASM (and that targeting them modulates airway reactivity). Comparison has been made with the way in which vagotomy (to reduce gastric acid secretion) was replaced by $\mathrm{H}_{2}$ antagonists and proton pump inhibitors. ${ }^{33}$ By analogy, if thermoplasty works by ablating proximal pacemakers, and if such pacemaker areas and/or cells can be specifically targeted, then we may similarly replace interventional procedures with pharmaceutical therapy.

\section{Postnatal REversion to Prenatal Phenotype (PRePP) hypothesis and airway homeokinesis}

Traditionally, it has been thought that prenatal ASM contractility is phasic while postnatal ASM is tonic. Nevertheless, the possibility remains that, in fact, asthma is a more phasic and oscillatory disease with essentially a postnatal reversion to prenatal phenotype (PRePP hypothesis) occurring in patients with asthma. Similarly, the postnatal airway has already been suggested to undergo rapid and continuous ASM-led reconfigurations that are maintained within tolerable limits in normal people ("homeokinesis"); such rapid reconfigurations appear to be exaggerated in people with asthma. ${ }^{34}$ Hence, asthma may be seen either as an abnormal return to phasic ASM contractility (albeit over longer oscillatory periods than in the prenatal lung) or as an aberration of postnatal ASM contractility whose normal oscillatory nature has been largely overlooked. Significant hindrance in evaluating these possibilities arises because explanted postnatal ASM appears always to require some form of stimulation to induce contraction (at least in the timescales that are feasible within the laboratory setting). Hence, prenatal ASM affords the unique opportunity to examine spontaneous contractility with potential bearing on ASM homeokinesis and postnatal airway configuration.

\section{CONCLUDING REMARKS}

Further investigation of ASM development before birth has been requested by the NHLBI. ${ }^{4}$ In this review we have examined the current knowledge on the development of ASM and explored the latest research findings that shed new light on both ASM development and function. Given that AP increases in frequency through gestation, the development of techniques to measure prenatal peristalsis in vivo may allow a novel test for lung maturity. Greater understanding of lung development may also permit the putative role of the fetal lung in the timing of parturition to be elucidated. ${ }^{11}$ Finally, given the resemblance between ASM and lymphangioleiomyomatosis cells and the contention that the latter may result from fetomaternal microchimerism, study of prenatal ASM may afford new insights into this rare but fascinating disease. ${ }^{35}$ New discoveries in the field of ASM development may therefore have the capacity to transform the screening for, and treatment of, a number of perinatal and postnatal lung diseases. In particular, identification of a postnatal airway pacemaker hierarchy that can be targeted selectively to treat asthma remains a tantalising possibility.

Acknowledgements: The author thanks the Academy of Medical Sciences, Health Foundation, Medical Research Council, Royal College of Surgeons of England, Birth Defects Foundation and Association of Laparoscopic Surgeons of Great Britain and Ireland for their support; and Professors Paul Losty, Dave Fernig, Mike White, David 
Warburton and Sue Wray; Dr Ted Burdyga, Dr Dave Spiller, Mr Neil Featherstone, Ms Gwen Connell and Ms Nikki Smith.

Competing interests: None.

\section{REFERENCES}

1. Mitzner W. Airway smooth muscle: the appendix of the lung. Am J Respir Crit Care Med 2004:169:787-90

2. Cox G, Thomson NC, Rubin AS, et al. Asthma control during the year after bronchial thermoplasty. N Engl J Med 2007;356:1327-37.

3. Schittny JC, Miserocchi G, Sparrow MP. Spontaneous peristaltic airway contractions propel lung liquid through the bronchial tree of intact and fetal lung explants. Am J Respir Cell Mol Biol 2000;23:11-8

4. Panettieri RA Jr, Kotlikoff MI, Gerthoffer WT, et al. Airway smooth muscle in bronchial tone, inflammation, and remodeling: basic knowledge to clinical relevance. Am J Respir Crit Care Med 2008;177:248-52

5. Holgate ST, Polosa R. The mechanisms, diagnosis, and management of severe asthma in adults. Lancet 2006;368:780-93.

6. Shi W, Bellusci S, Warburton D. Lung development and adult lung diseases. Chest 2007; 132:651-6.

7. Muratore CS, Kharasch V, Lund DP, et al. Pulmonary morbidity in 100 survivors of congenital diaphragmatic hernia monitored in a multidisciplinary clinic. J Pediatr Surg 2001;36:133-40.

8. Yang Y, Beqaj S, Kemp $P$, et al. Stretch-induced alternative splicing of serum response factor promotes bronchial myogenesis and is defective in lung hypoplasia. $J$ Clin Invest 2000;106:1321-30.

9. Jesudason EC. Small lungs and suspect smooth muscle: congenital diaphragmatic hernia and the smooth muscle hypothesis. J Pediatr Surg 2006;41:431-5.

10. Kooyman G, Ponganis P. The physiological basis of diving to depth: birds and mammals. Annu Rev Physiol 1998;60:19-32.

11. Jesudason EC. Exploiting mechanical stimuli to rescue growth of the hypoplastic lung. Pediatr Surg Int 2007;23:827-36.

12. Jesudason EC, Smith NP, Connell MG, et al. Developing rat lung has a sided pacemaker region for morphogenesis-related airway peristalsis. Am J Respir Cell Mol Biol 2005; 32:118-27.

13. Yang $\mathbf{Y}$, Palmer $\mathrm{KC}$, Relan $\mathrm{N}$, et al. Role of laminin polymerization at the epithelia mesenchymal interface in bronchial myogenesis. Development 1998;125:2621-9.

14. Yang Y, Relan NK, Przywara DA, et al. Embryonic mesenchymal cells share the potential for smooth muscle differentiation: myogenesis is controlled by the cell's shape. Development 1999;126:3027-33.

15. Beqaj S, Jakkaraju S, Mattingly RR, et al. High RhoA activity maintains the undifferentiated mesenchymal cell phenotype, whereas RhoA down-regulation by laminin-2 induces smooth muscle myogenesis. J Cell Biol 2002;156:893-903.

16. Harding R, Hooper SB. Regulation of lung expansion and lung growth before birth J Appl Physiol 1996;81:209-24.

17. McCray PB Jr. Spontaneous contractility of human fetal airway smooth muscle. Am J Respir Cell Mol Biol 1993;8:573-80.
18. Parvez 0, Voss AM, de Kok M, et al. Bronchial muscle peristaltic activity in the fetal rat. Pediatr Res 2006;59:756-61.

19. Pandya HC, Innes J, Hodge R, et al. Spontaneous contraction of pseudoglandularstage human airspaces is associated with the presence of smooth muscle-alpha-actin and smooth muscle-specific myosin heavy chain in recently differentiated fetal human airway smooth muscle. Biol Neonate 2006;89:211-9.

20. Unbekandt $\mathbf{M}$, del Moral P, Sala F, et al. Tracheal occlusion increases the rate of epithelial branching of embryonic mouse lung via the FGF10-FGFR2b-Sprouty2 pathway. Mech Dev 2008;125:314-24.

21. Mailleux AA, Kelly R, Veltmaat JM, et al. Fgf10 expression identifies parabronchial smooth muscle cell progenitors and is required for their entry into the smooth muscle cell lineage. Development 2005:132:2157-66.

22. Min H, Danilenko DM, Scully SA, et al. Fgf-10 is required for both limb and lung development and exhibits striking functional similarity to Drosophila branchless. Genes Dev 1998;12:3156-61.

23. Ghabrial A, Luschnig S, Metzstein MM, et al. Branching morphogenesis of the Drosophila tracheal system. Annu Rev Cell Dev Biol 2003;19:623-47.

24. Shan L, Subramaniam M, Emanuel R, et al. Centrifugal migration of mesenchymal cells in embryonic lung. Dev Dyn 2008;237:750-7.

25. Jesudason EC. Smith NP, Connell MG, et al. Peristalsis of airway smooth muscle is developmentally regulated and uncoupled from hypoplastic lung growth. Am J Physiol Lung Cell Mol Physiol 2006;291:L559-65.

26. Featherstone NC, Jesudason EC, Connell MG, et al. Spontaneous propagating calcium waves underpin airway peristalsis in embryonic rat lung. Am J Respir Cell Mol Biol 2005;33:153-60.

27. Featherstone NC, Connell MG, Fernig DG, et al. Airway smooth muscle dysfunction precedes teratogenic congenital diaphragmatic hernia and may contribute to hypoplastic lung morphogenesis. Am J Respir Cell Mol Biol 2006;35:571-8.

28. Featherstone NC, Connell MG, Fernig DG, et al. Airway peristalsis: a novel regulator of chloride-led lung liquid production? (abstract). Am J Respir Crit Care Med 2008:177:A315.

29. Olver RE, Walters DV, S MW. Developmental regulation of lung liquid transport Annu Rev Physiol 2004:66:77-101.

30. Harrison MR, Keller RL, Hawgood SB, et al. A randomized trial of fetal endoscopic tracheal occlusion for severe fetal congenital diaphragmatic hernia. N Engl J Med 2003:349:1916-24.

31. Ba'ath ME, Jesudason EC, Losty PD. How useful is the lung-to-head ratio in predicting outcome in the fetus with congenital diaphragmatic hernia? A systematic review and meta-analysis. Ultrasound Obstet Gynecol 2007;30:897-906.

32. Nelson SM, Hajivassiliou CA, Haddock G, et al. Rescue of the hypoplastic lung by prenatal cyclical strain. Am J Respir Crit Care Med 2005:171:1395-402.

33. Solway J, Irvin CG. Airway smooth muscle as a target for asthma therapy. N Engl J Med 2007;356:1367-9.

34. Que CL, Kenyon CM, Olivenstein R, et al. Homeokinesis and short-term variability of human airway caliber. J Appl Physiol 2001;91:1131-41.

35. Krymskaya VP. Smooth muscle-like cells in pulmonary lymphangioleiomyomatosis Proc Am Thorac Soc 2008:5:119-26.

\section{Access all our original articles online even before they appear in a print issue!}

Online First is an exciting innovation that allows the latest clinical research papers to go from acceptance to your browser within days, keeping you at the cutting edge of medicine.

Simply follow the Online First link on the homepage and read the latest Online First articles that are available as unedited manuscripts in downloadable PDF form. The articles are peer reviewed, accepted for publication and indexed by PubMed but not yet included in a journal issue, so you'll be among the first to read them! 